University of Nebraska - Lincoln

DigitalCommons@University of Nebraska - Lincoln

USDA Forest Service / UNL Faculty Publications U.S. Department of Agriculture: Forest Service -National Agroforestry Center

2012

\title{
Tree and impervious cover change in U.S. cities
}

David J. Nowak

USDA Forest Service, dnowak@fs.fed.us

Eric J. Greenfield

USDA Forest Service, ejgreenfield@fs.fed.us

Follow this and additional works at: https://digitalcommons.unl.edu/usdafsfacpub

Nowak, David J. and Greenfield, Eric J., "Tree and impervious cover change in U.S. cities" (2012). USDA Forest Service / UNL Faculty Publications. 240.

https://digitalcommons.unl.edu/usdafsfacpub/240

This Article is brought to you for free and open access by the U.S. Department of Agriculture: Forest Service -National Agroforestry Center at DigitalCommons@University of Nebraska - Lincoln. It has been accepted for inclusion in USDA Forest Service / UNL Faculty Publications by an authorized administrator of DigitalCommons@University of Nebraska - Lincoln. 


\title{
Tree and impervious cover change in U.S. cities
}

\author{
David J. Nowak*, Eric J. Greenfield \\ USDA Forest Service, Northern Research Station, 5 Moon Library, SUNY-ESF, Syracuse, NY 13210, United States
}

\section{A R T I C L E I N F O}

\section{Keywords:}

City trees

Ecosystem services

Forest monitoring

Urban forestry

Urban greening

Urban trees

\begin{abstract}
A B S T R A C T
Paired aerial photographs were interpreted to assess recent changes in tree, impervious and other cover types in 20 U.S. cities as well as urban land within the conterminous United States. National results indicate that tree cover in urban areas of the United States is on the decline at a rate of about $7900 \mathrm{ha} / \mathrm{yr}$ or 4.0 million trees per year. Tree cover in 17 of the 20 analyzed cities had statistically significant declines in tree cover, while 16 cities had statistically significant increases in impervious cover. Only one city (Syracuse, NY) had a statistically significant increase in tree cover. City tree cover was reduced, on average, by about 0.27 percent/yr, while impervious surfaces increased at an average rate of about 0.31 percent/yr. As tree cover provides a simple means to assess the magnitude of the overall urban forest resource, monitoring of tree cover changes is important to understand how tree cover and various environmental benefits derived from the trees may be changing. Photo-interpretation of digital aerial images can provide a simple and timely means to assess urban tree cover change to help cities monitor progress in sustaining desired urban tree cover levels.
\end{abstract}

Published by Elsevier GmbH.

\section{Introduction}

Tree cover in cities is constantly changing due to various natural and anthropogenic forces. Natural forces for change include natural regeneration, tree growth and tree mortality from insects and diseases or old age. Anthropogenic factors that influence tree cover include tree planting and tree mortality or removal from either direct or indirect human actions such as development and air pollution (Nowak, 1993). The combination of these factors through time determines existing and future tree cover levels.

An important question for city managers is how their local tree cover is currently changing as present-day benefits derived from urban forests are related to the amount of tree cover in cities. As many urban forest ecosystem services are directly related to the amount of healthy and functioning leaves, tree cover becomes a simple measure of the extent of the urban forest and consequently the magnitude of services provided by the forest. To help sustain tree cover in cities, various city programs are planting large numbers of trees (e.g., City of New York, 2011; City of Los Angeles, 2011), protecting existing trees (e.g., Town of Chapel Hill, 2011; City of Pasadena, 2011) and developing tree canopy goals (e.g., City of Seattle, 2011; Maryland Department of Natural Resources, 2011).

Though tree cover in cities is constantly changing, limited studies have investigated how overall tree cover in cities has or is

\footnotetext{
* Corresponding author. Tel.: +1 315448 3212; fax: +1 3154483216. E-mail addresses: dnowak@fs.fed.us (D.J. Nowak), ejgreenfield@fs.fed.us (E.J. Greenfield).
}

changing. Nowak (1993) illustrated through an analysis of historical imagery and documents that the tree cover in Oakland, CA, has increased from a presettlement tree cover of approximately 2 percent in 1850s to 19 percent in 1991. Land cover maps have been used to quantify how various cover classes have changed through time, but assessments of tree cover change within cities are limited (e.g., Zhou et al., 2008). In Seattle, tree cover was estimated to change from 22.5 percent in 2002 to 22.9 percent in 2007 by comparing digital land cover maps developed from $0.6 \mathrm{~m}$ resolution imagery (Parlin, 2009). However, the accuracy of the map classification is unknown and comparing cover maps to estimate change can lead to false changes due to misclassification of cover types on either map.

Various land cover change analyses have been conducted using satellite-based approaches. Moderate Resolution Imaging Spectroradiometer (MODIS) data (250-m) and Landsat data (30-m) have and are being used to estimate changes in land cover and impervious surface cover (e.g., Yang et al., 2003; Lunetta et al., 2006; U.S. EPA, 2011). MODIS data (500-m) also has the ability to estimate change in percent tree cover across the globe (Hansen et al., 2003; Schwarz et al., 2006). These satellite-based approaches have limitations based on image resolution and inaccuracies of image classifications. Photo-interpretation of high resolution images to detect cover changes has the ability to overcome these limitations, but lacks the ability to develop detailed comprehensive cover change maps.

Trees and impervious surfaces provide numerous ecosystem services and values to a community, but also have various economic or environmental costs. Trees provide various benefits associated 
with air and water quality, building energy conservation, cooler air temperatures, reductions in ultraviolet radiation, and many other environmental and social benefits (e.g., Dwyer et al., 1992; Kuo and Sullivan, 2001; Westphal, 2003; Wolf, 2003; Nowak and Dwyer, 2007). Costs associated with trees are both economic (e.g., planting and maintenance and increased building energy costs) and environmental (e.g., pollen and volatile organic compound emissions) (Nowak and Dwyer, 2007).

Likewise, impervious cover plays an important role in the landscape, particularly in urban areas. These surfaces, such as roads, buildings, sidewalks, and parking lots, facilitate transportation and provide shelter, but also can negatively impact the environment. Increased impervious surfaces enhance local temperatures and heat islands (Oke, 1989; Heisler and Brazel, 2010), which consequently affects building energy use, human comfort and health, ozone production, and pollutant emissions in cities. In addition, impervious surfaces significantly affect urban hydrology (e.g., stream flow and water quality) (e.g., U.S. EPA, 1983; National Research Council, 2008).

As development occurs in forests, tree cover will decrease to make space for buildings and other impervious surfaces. In non-forest regions, tree cover can increase due to urbanization (unpublished data). Thus, urbanization as a process will alter regional tree cover. As tree cover changes in cities, so will the associated ecosystem services and their effects on environmental quality and human health. Unfortunately, within existing cities, rates and direction of change in tree and impervious cover are largely unknown. This paper investigates tree and impervious cover change in urban areas and select cities across the United States using a simple and repeatable measure that can be used worldwide where paired multi-year digital aerial imagery exists. The objective of this paper is to determine the current direction and rate of tree and impervious cover change in U.S. cities to help guide cities in sustaining desired tree cover levels and associated ecosystem services.

\section{Methods}

To determine the percent tree/shrub cover (hereafter referred to as tree cover or canopy) and impervious cover change in cities in the United States, 20 cities from across the nation were selected
(Table 1). Some cities were selected based on existing projects (Syracuse, NY; Baltimore, MD; Spokane, WA). Other cities were selected by picking major cities scattered throughout the conterminous United States where paired imagery could be obtained. Two cities were specifically selected to determine the effect of recent suspected tree cover change: (1) New Orleans, LA (effect of 2005 Hurricane Katrina), and (2) Detroit, MI (effect of recent infestation of emerald ash borer (Agrilus planipennis)). For each city, paired digital aerial photographs were obtained for the most recent date possible and imagery as close to $5 \mathrm{yr}$ prior to the most current date as possible.

In 18 of the 20 cities, 1000 random points were laid and interpreted across the city to provide a maximum standard error of 1.6 percent if all points are classified (Lindgren and McElrath, 1969). In two cities, more points were laid and interpreted (Baltimore: 2500 points; Spokane, WA: 2000 points). City geographic boundaries were determined using census incorporated or designated places boundaries (U.S. Census Bureau, 2007). Each point was laid in the same geographic position on both sets of temporal images in the city, and paired image interpretation was conducted (i.e., interpreter classified each point pair by contrasting and classifying the image points in sequence). In cases of misregistration of the image or point, the interpreter corrected the point location to ensure the exact same location was interpreted. For example, sometimes the points would shift position slightly between images due to issues of image misregistration. In these cases, the interpreter moved the point on the most recent image back to the position on the oldest image to make the interpretation of change at the same point on both images.

In some cases, not all of the points could be classified. Nonclassification occurred when one of the images were missing part of the city area (incomplete imagery) or had cloud cover. All cities had greater than 97.2 percent of the points interpreted. As some cities have substantial amounts of water within their city boundary (Table 1), cover estimates were only based on points that were not classified as water in both years. That is, permanent water points were deleted from the sample so that cover estimates were based on city land area, not city total area.

For the photo-interpretation, trained photo interpreters with experience interpreting leaf-off and leaf-on imagery classified each point as to either: trees/shrubs (woody vegetation), grass

Table 1

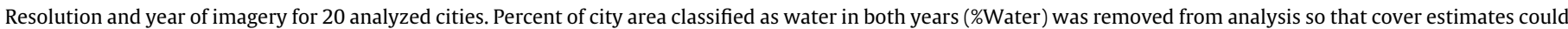
be based on land area. Human population density change (\#/ha) between year 1 and year 2 is based on U.S. Census estimates (1).

\begin{tabular}{|c|c|c|c|c|c|c|c|c|c|c|}
\hline City & Year 1 & $\operatorname{Res}^{\mathrm{a}}(\mathrm{m})$ & Leaf on/off & Year 2 & Res. $^{\mathrm{a}}(\mathrm{m})$ & Leaf on/off & $\%$ interp $^{b}$ & \%Water & $n$ & Change (\#/ha) \\
\hline Albuquerque, NM & 2006 & 0.15 & Off & 2009 & 1 & On & 100 & 0.2 & 998 & 0.6 \\
\hline Atlanta, GA & 2005 & 2 & On & 2009 & 1 & On & 99.5 & 0.4 & 991 & 1.7 \\
\hline Baltimore, MD & 2001 & 1 & On & 2005 & 1 & On & 99.9 & 12.6 & 2184 & -0.2 \\
\hline Boston, MA & 2003 & 1 & On & 2008 & 1 & On & 99.9 & 13.6 & 863 & 2.3 \\
\hline Chicago, IL & 2005 & 2 & On & 2009 & 1 & On & 100 & 0.8 & 992 & 0.5 \\
\hline Denver, CO & 2005 & 1 & On & 2009 & 1 & On & 100 & 1.6 & 984 & 1.2 \\
\hline Detroit, MI & 2005 & 1 & On & 2009 & 1 & On & 99.9 & 0.3 & 996 & -0.3 \\
\hline Houston, TX & 2004 & 1 & On & 2009 & 1 & On & 99.5 & 1.6 & 979 & 1.4 \\
\hline Kansas City, MO & 2003 & 1 & On & 2009 & 1 & On & 100 & 1.5 & 985 & 0.4 \\
\hline Los Angeles, CA & 2005 & 1 & On & 2009 & 1 & On & 100 & 0.2 & 998 & 0.3 \\
\hline Miami, FL & 2003 & 1 & On & 2009 & 0.3 & On & 100 & 9.3 & 907 & 6.3 \\
\hline Minneapolis, MN & 2003 & 1 & On & 2008 & 1 & On & 98.9 & 7.1 & 919 & 0.3 \\
\hline Nashville, TN & 2003 & 0.15 & Off & 2008 & 0.15 & Off & 100 & 0.7 & 993 & 0.3 \\
\hline New Orleans, LA & 2005 & 2 & On & 2009 & 1 & On & 97.2 & 38.4 & 563 & -2.1 \\
\hline New York, NY & 2004 & 0.15 & On & 2009 & 1 & On & 98.1 & 2.9 & 953 & 2.8 \\
\hline Pittsburgh, PA & 2004 & 1 & On & 2008 & 1 & On & 99.5 & 4.8 & 947 & -0.6 \\
\hline Portland, OR & 2005 & 1 & On & 2009 & 1 & On & 100 & 1.6 & 984 & 1.0 \\
\hline Spokane, WA & 2002 & 0.15 & On & 2007 & 0.15 & On & 100 & 1.0 & 1980 & 0.3 \\
\hline Syracuse, NY & 2003 & 0.3 & Off & 2009 & 0.3 & Off & 99.6 & 2.0 & 976 & -0.7 \\
\hline Tacoma, WA & 2001 & 0.15 & On & 2005 & 0.15 & On & 100 & 8.6 & 914 & -0.1 \\
\hline
\end{tabular}

a Image (pixel) resolution.

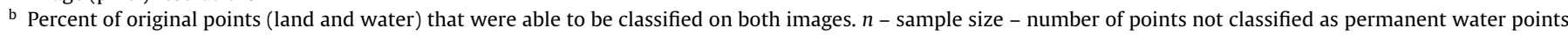
(classified as water in both years). 
or herbaceous cover, bare soil, water, impervious (buildings), impervious (roads), or impervious (other). For the analysis of Albuquerque, NM, only, an eighth class of scrub/shrub was added due to the different vegetation cover morphology of that region. This class was included in the tree/shrub cover classification, but the scrub/shrub class results were also reported separately. Within Syracuse, which was one of the first cities analyzed, impervious other and impervious road categories were combined by the interpreter as was the grass/herbaceous and soil categories. In subsequent city analyses these categories were separated.

In interpreting change from aerial imagery, image parallax (tall objects appearing to lean on the image) and seasonal changes can appear to cause changes, but in fact are not actual changes. In these cases the interpreter could use judgment to determine if actual change did occur. In cases of tall object parallax, the interpreter's classification was based on the oldest image and if there was no change, both dates of imagery were classified the same. For example, tall objects (e.g., buildings and trees) may lean to the left in the first image, but lean to the right in the second image and a point may land on the object in the first image, but miss the object in the second image. The point classification would appear to change class, but no actual change would have occurred. Also agricultural fields can change cover class depending on time of year (herbaceous cover vs. bare soil depending upon time of imagery). These types of seasonal changes were classified as no change and classified as herbaceous cover. By conducting paired-point image analysis, the interpreter can correct these false changes to no change in the analysis. A five-percent random sample of points was reinterpreted by another photo-interpreter to check for classification accuracy. Overall, the two interpreters were in agreement on 97 percent of the classifications.

Within each city, the percentage of each cover class $(p)$ was calculated as the number of sample points $(x)$ hitting the cover attribute divided by the total number of interpretable sample points $(n)$ within the area of analysis $(p=x / n)$. The standard error of the estimate $(S E)$ was calculated as $S E=\sqrt{p \times(1-p) / n}$ (Lindgren and McElrath, 1969). This method has been used to assess canopy cover in many cities (e.g., Nowak et al., 1996).

If changes in cover classes were observed at any point on the image then it is known that cover classes are changing within the city (i.e., no statistical test is needed to determine if change is greater than zero). However, as a cover class can both gain and lose cover through time and space, the McNemar test (Sokal and Rohlf, 2003) was used to determine if the net change in cover was different from zero (alpha levels 0.90 and 0.95 ). Pearson product moment correlation was used to test for a relationship between change in percent tree cover and change in population density among the 18 cities.

As the overall time frame of change in cover varied among cities from between 3 and $6 \mathrm{yr}$, change results were annualized for comparative purposes among cities. Results were combined with city area and population data from the year of the oldest photo date (U.S. Census Bureau, 2011) to determine actual tree and impervious cover change (ha) and cover change per capita in each city. Results of percent change were reported as absolute change (percent of city area that changed = cover change/city area) and relative change (percent of existing cover class that changed = cover change/original cover area). For example, a city with 30 percent tree cover that changed to 20 percent tree cover would have a 10 percent absolute change, but a 33 percent relative change.

As the 20 analyzed cities are not a truly random sample, an analysis of change in tree and impervious cover in urban areas across the conterminous United States was conducted using Google Earth ${ }^{\circledR}$ (Google, 2011) imagery to determine the relative magnitude of net change in urban tree and impervious cover. Urban land was defined based on population density as delimited using the U.S. Census
Bureau's (2007) definition: all territory, population, and housing units located within urbanized areas or urban clusters. Urbanized area and urban cluster boundaries encompass densely settled territories, which are described by one of the following:

- one or more block groups or census blocks with a population density of at least 386.1 people $/ \mathrm{km}^{2}$ (1000 people/mile ${ }^{2}$ ),

- surrounding census blocks with a minimum population density of 193.1 people $/ \mathrm{km}^{2}$ (500 people/mile $\mathrm{m}^{2}$ ), or

- less densely settled blocks that form enclaves or indentations, or are used to connect discontinuous areas.

In the conterminous United States, 1000 points randomly located within urban land were interpreted based on paired imagery from Google using the images with the most recent date and the next oldest interpretable imagery with the goal of trying to get the second set of imagery about $5 \mathrm{yr}$ apart from the first set. Imagery date along with cover class was recorded for each point. This type of analysis of change with Google imagery has varying date issues that were not encountered with the paired city imagery, but does give a general indication of direction and magnitude of change nationally. Analysis of Google imagery was similar to the city imagery in terms of non-interpretable images and adjusting for misregistered images. However, Google imagery could also not be interpreted in some locations due to poor image resolution. Overall, 97 percent of the points could be interpreted using Google imagery.

\section{Results}

Of the 20 cities analyzed, tree cover ranged from 53.9 percent in Atlanta to 9.6 percent in Denver; building impervious cover ranged from 27.1 percent in Chicago to 4.8 percent in Kansas City; road and other impervious cover ranged from 36.2 percent in Miami to 12.3 percent in Nashville; and total impervious cover varied from 61.1 percent in New York City to 17.7 percent in Nashville (Table 2). Two cover classes - tree/shrub and bare soil generally exhibited a reduction in percent cover, while the other land classes generally exhibited an increase in cover.

Change in tree cover during the varying periods of analysis ranged from reduction in percent tree cover of -9.6 in New Orleans to an increase in percent tree cover of 1.0 in Syracuse (Table 3). Nineteen of the 20 cities analyzed showed a reduction in tree cover, 17 of those cities had a statistically significant net reduction. Average change was calculated for all 20 cities and for 18 cities - excluding the two cities (New Orleans and Detroit) that were targeted due to an expected loss in tree cover. Percent tree cover dropped on average by 1.1 percent during the varying periods of analysis ( 1.5 percent for 20 city average) with the greatest decreases in percent tree cover in New Orleans ( -9.6 percent), Houston ( -3.0 percent) and Albuquerque ( -2.7 percent). The relative reduction in tree cover was as high as -29.2 percent in New Orleans, but averaged -3.8 percent $(-5.0$ percent for 20 city average).

Cities with the greatest annual loss in tree cover were New Orleans (average of $-1120 \mathrm{ha} / \mathrm{yr}$ ), Houston ( $-890 \mathrm{ha} / \mathrm{yr}$ ) and Albuquerque $(-420 \mathrm{ha} / \mathrm{yr})$ (Table 3 ). Tree cover losses per capita were greatest in New Orleans $\left(-24.6 \mathrm{~m}^{2} /\right.$ person/yr), Albuquerque $\left(-8.3 \mathrm{~m}^{2} /\right.$ person/yr) and Nashville $\left(-5.3 \mathrm{~m}^{2} /\right.$ person/yr $)$ with an average loss of $-1.9 \mathrm{~m}^{2} /$ person $/ \mathrm{yr}\left(-3.0 \mathrm{~m}^{2} /\right.$ person/yr for 20 city average). Average annual loss in percent tree cover was -0.27 percent/yr ( -0.37 percent/yr for 20 city average). Relative annual loss in tree cover was -0.90 percent/yr $(-1.29$ percent/yr for 20 city average). Loss of tree cover was slightly correlated to increased population density in the 18 cities (Pearson product moment correlation coefficient $(r)=-0.31$ ). 
Table 2

Change of percent of city land area occupied by various cover classes in 20 U.S. cities.

\begin{tabular}{|c|c|c|c|c|c|c|c|c|c|c|}
\hline \multirow[t]{2}{*}{ City } & \multirow[t]{2}{*}{1 st year cover class } & \multicolumn{7}{|c|}{ 2nd year cover class } & \multicolumn{2}{|c|}{ 1st year } \\
\hline & & Grass/herb ${ }^{a}$ & Tree/shrub & Imp. bldgb & Imp. road $^{c}$ & Imp. other ${ }^{\mathrm{d}}$ & Water & Soil & Total & SE \\
\hline \multirow[t]{10}{*}{ Albuquerque, NM (2006-2009)e } & Grass/herb & 8.8 & 0.1 & 0.1 & 0.0 & 0.1 & 0.0 & 0.0 & 9.1 & 0.9 \\
\hline & Tree/shrub & 0.4 & 38.0 & 0.0 & 0.0 & 0.4 & 0.0 & 2.0 & 40.8 & 1.6 \\
\hline & Imp. bldg & 0.1 & 0.0 & 11.9 & 0.0 & 0.0 & 0.0 & 0.0 & 12.0 & 1.0 \\
\hline & Imp. road & 0.0 & 0.0 & 0.0 & 9.4 & 0.0 & 0.0 & 0.0 & 9.4 & 0.9 \\
\hline & Imp. other & 0.0 & 0.0 & 0.0 & 0.0 & 13.9 & 0.0 & 0.0 & 13.9 & 1.1 \\
\hline & Water & 0.0 & 0.0 & 0.0 & 0.0 & 0.0 & 0.0 & 0.0 & 0.0 & 0.0 \\
\hline & Soil & 0.4 & 0.0 & 0.5 & 0.3 & 0.5 & 0.0 & 13.0 & 14.7 & 1.1 \\
\hline & 2nd year total & 9.7 & 38.1 & 12.5 & 9.7 & 14.9 & 0.0 & 15.0 & & \\
\hline & 2nd year SE & 0.9 & 1.5 & 1.0 & 0.9 & 1.1 & 0.0 & 1.1 & & \\
\hline & Net (2006-2009) & 0.6 & -2.7 & 0.5 & 0.3 & 1.0 & 0.0 & 0.3 & & \\
\hline \multirow[t]{10}{*}{ Atlanta, GA (2005-2009) } & Grass/herb & 15.1 & 0.4 & 0.1 & 0.0 & 0.3 & 0.0 & 0.6 & 16.5 & 1.2 \\
\hline & Tree/shrub & 1.0 & 51.6 & 0.4 & 0.1 & 0.3 & 0.0 & 0.5 & 53.9 & 1.6 \\
\hline & Imp. bldg & 0.0 & 0.0 & 9.6 & 0.0 & 0.1 & 0.0 & 0.1 & 9.8 & 0.9 \\
\hline & Imp. road & 0.0 & 0.0 & 0.0 & 7.4 & 0.0 & 0.0 & 0.0 & 7.4 & 0.8 \\
\hline & Imp. other & 0.0 & 0.0 & 0.1 & 0.0 & 9.2 & 0.0 & 0.0 & 9.3 & 0.9 \\
\hline & Water & 0.0 & 0.0 & 0.0 & 0.0 & 0.0 & 0.0 & 0.0 & 0.0 & 0.0 \\
\hline & Soil & 1.3 & 0.1 & 0.2 & 0.2 & 0.2 & 0.0 & 1.1 & 3.1 & 0.6 \\
\hline & 2nd year total & 17.5 & 52.1 & 10.4 & 7.7 & 10.1 & 0.0 & 2.3 & & \\
\hline & 2nd year SE & 1.2 & 1.6 & 1.0 & 0.8 & 1.0 & 0.0 & 0.5 & & \\
\hline & Net (2005-2009) & 0.9 & -1.8 & 0.6 & 0.3 & 0.8 & 0.0 & -0.8 & & \\
\hline \multirow[t]{10}{*}{ Baltimore, MD (2001-2005) } & Grass/herb & 22.2 & 0.1 & 0.2 & 0.0 & 0.7 & 0.0 & 0.3 & 23.5 & 0.9 \\
\hline & Tree/shrub & 0.9 & 28.4 & 0.4 & 0.1 & 0.5 & 0.0 & 0.1 & 30.4 & 1.0 \\
\hline & Imp. bldg & 0.0 & 0.0 & 15.3 & 0.0 & 0.0 & 0.0 & 0.3 & 15.6 & 0.8 \\
\hline & Imp. road & 0.0 & 0.0 & 0.0 & 10.9 & 0.0 & 0.0 & 0.0 & 11.0 & 0.7 \\
\hline & Imp. other & 0.0 & 0.0 & 0.0 & 0.0 & 16.8 & 0.0 & 0.2 & 17.1 & 0.8 \\
\hline & Water & 0.0 & 0.0 & 0.0 & 0.0 & 0.0 & 0.0 & 0.0 & 0.0 & 0.0 \\
\hline & Soil & 0.1 & 0.0 & 0.3 & 0.0 & 0.3 & 0.0 & 1.6 & 2.3 & 0.3 \\
\hline & 2nd year total & 23.2 & 28.5 & 16.3 & 11.0 & 18.5 & 0.0 & 2.5 & & \\
\hline & 2nd year SE & 0.9 & 1.0 & 0.8 & 0.7 & 0.8 & 0.0 & 0.3 & & \\
\hline & Net (2001-2005) & -0.4 & -1.9 & 0.7 & 0.0 & 1.3 & 0.0 & 0.2 & & \\
\hline \multirow[t]{10}{*}{ Boston, MA (2003-2008) } & Grass/herb & 17.8 & 0.5 & 0.1 & 0.0 & 0.6 & 0.0 & 0.1 & 19.1 & 1.3 \\
\hline & Tree/shrub & 0.6 & 27.1 & 0.6 & 0.2 & 0.1 & 0.0 & 0.2 & 28.9 & 1.5 \\
\hline & Imp. bldg & 0.1 & 0.0 & 16.5 & 0.0 & 0.1 & 0.0 & 0.0 & 16.7 & 1.3 \\
\hline & Imp. road & 0.0 & 0.0 & 0.0 & 12.5 & 0.0 & 0.0 & 0.0 & 12.5 & 1.1 \\
\hline & Imp. other & 0.2 & 0.2 & 0.0 & 0.1 & 18.4 & 0.0 & 0.0 & 19.0 & 1.3 \\
\hline & Water & 0.1 & 0.0 & 0.0 & 0.0 & 0.0 & 0.0 & 0.7 & 0.8 & 0.3 \\
\hline & Soil & 0.5 & 0.1 & 0.1 & 0.1 & 0.5 & 0.0 & 1.7 & 3.0 & 0.6 \\
\hline & 2nd year total & 19.4 & 27.9 & 17.3 & 13.0 & 19.7 & 0.0 & 2.8 & & \\
\hline & 2nd year SE & 1.3 & 1.5 & 1.3 & 1.1 & 1.4 & 0.0 & 0.6 & & \\
\hline & Net (2003-2008) & 0.2 & -0.9 & 0.6 & 0.5 & 0.7 & -0.8 & -0.2 & & \\
\hline \multirow[t]{10}{*}{ Chicago, IL (2005-2009) } & Grass/herb & 20.0 & 0.0 & 0.0 & 0.0 & 0.3 & 0.1 & 0.4 & 20.8 & 1.3 \\
\hline & Tree/shrub & 0.3 & 18.0 & 0.1 & 0.0 & 0.0 & 0.0 & 0.1 & 18.5 & 1.2 \\
\hline & Imp. bldg & 0.4 & 0.0 & 26.5 & 0.0 & 0.1 & 0.0 & 0.1 & 27.1 & 1.4 \\
\hline & Imp. road & 0.0 & 0.0 & 0.0 & 12.1 & 0.0 & 0.0 & 0.0 & 12.1 & 1.0 \\
\hline & Imp. other & 0.0 & 0.0 & 0.2 & 0.0 & 19.1 & 0.0 & 0.0 & 19.3 & 1.3 \\
\hline & Water & 0.0 & 0.0 & 0.0 & 0.0 & 0.0 & 0.0 & 0.0 & 0.0 & 0.0 \\
\hline & Soil & 0.0 & 0.0 & 0.0 & 0.0 & 0.1 & 0.1 & 2.0 & 2.2 & 0.5 \\
\hline & 2nd year total & 20.7 & 18.0 & 26.8 & 12.1 & 19.6 & 0.2 & 2.6 & & \\
\hline & 2nd year SE & 1.3 & 1.2 & 1.4 & 1.0 & 1.3 & 0.1 & 0.5 & & \\
\hline & Net (2005-2009) & -0.1 & -0.5 & -0.3 & 0.0 & 0.3 & 0.2 & 0.4 & & \\
\hline \multirow[t]{10}{*}{ Denver, CO (2005-2009) } & Grass/herb & 41.1 & 0.0 & 0.1 & 0.0 & 0.3 & 0.0 & 0.9 & 42.4 & 1.6 \\
\hline & Tree/shrub & 0.1 & 9.6 & 0.1 & 0.0 & 0.1 & 0.0 & 0.0 & 9.9 & 1.0 \\
\hline & Imp. bldg & 0.0 & 0.0 & 12.8 & 0.0 & 0.0 & 0.0 & 0.1 & 12.9 & 1.1 \\
\hline & Imp. road & 0.0 & 0.0 & 0.1 & 12.5 & 0.0 & 0.0 & 0.0 & 12.6 & 1.1 \\
\hline & Imp. other & 0.2 & 0.0 & 0.2 & 0.1 & 13.9 & 0.0 & 0.1 & 14.5 & 1.1 \\
\hline & Water & 0.0 & 0.0 & 0.0 & 0.0 & 0.0 & 0.0 & 0.0 & 0.0 & 0.0 \\
\hline & Soil & 0.8 & 0.0 & 0.1 & 0.1 & 1.0 & 0.2 & 5.5 & 7.7 & 0.9 \\
\hline & 2nd year total & 42.2 & 9.6 & 13.4 & 12.7 & 15.3 & 0.2 & 6.6 & & \\
\hline & 2nd year SE & 1.6 & 0.9 & 1.1 & 1.1 & 1.1 & 0.1 & 0.8 & & \\
\hline & Net (2005-2009) & -0.2 & -0.3 & 0.5 & 0.1 & 0.8 & 0.2 & -1.1 & & \\
\hline \multirow[t]{4}{*}{ Detroit, MI (2005-2009) } & Grass/herb & 27.9 & 0.1 & 0.0 & 0.0 & 0.2 & 0.0 & 0.3 & 28.5 & 1.4 \\
\hline & Tree/shrub & 0.1 & 22.3 & 0.1 & 0.2 & 0.4 & 0.0 & 0.1 & 23.2 & 1.3 \\
\hline & Imp. bldg & 0.1 & 0.0 & 17.1 & 0.0 & 0.0 & 0.0 & 0.0 & 17.2 & 1.2 \\
\hline & Imp. road & 0.0 & 0.0 & 0.0 & 14.7 & 0.0 & 0.0 & 0.0 & 14.7 & 1.1 \\
\hline
\end{tabular}


Table 2 (Continued)

\begin{tabular}{|c|c|c|c|c|c|c|c|c|c|c|}
\hline \multirow[t]{2}{*}{ City } & \multirow[t]{2}{*}{1 st year cover class } & \multicolumn{7}{|c|}{ 2nd year cover class } & \multicolumn{2}{|c|}{ 1st year } \\
\hline & & Grass/herb ${ }^{a}$ & Tree/shrub & Imp. bldgb & Imp. roadc & Imp. other ${ }^{\mathrm{d}}$ & Water & Soil & Total & SE \\
\hline & Imp. other & 0.0 & 0.0 & 0.1 & 0.0 & 14.5 & 0.0 & 0.0 & 14.6 & 1.1 \\
\hline & Water & 0.0 & 0.0 & 0.0 & 0.0 & 0.0 & 0.0 & 0.0 & 0.0 & 0.0 \\
\hline & Soil & 0.2 & 0.1 & 0.1 & 0.0 & 0.3 & 0.0 & 1.2 & 1.9 & 0.4 \\
\hline & 2nd year total & 28.3 & 22.5 & 17.4 & 14.9 & 15.4 & 0.0 & 1.6 & & \\
\hline & 2nd year SE & 1.4 & 1.3 & 1.2 & 1.1 & 1.1 & 0.0 & 0.4 & & \\
\hline & Net (2005-2009) & -0.2 & -0.7 & 0.2 & 0.2 & 0.8 & 0.0 & -0.3 & & \\
\hline \multirow[t]{10}{*}{ Houston, TX (2004-2009) } & Grass/herb & 28.7 & 0.0 & 0.5 & 0.0 & 0.5 & 0.2 & 0.2 & 30.1 & 1.5 \\
\hline & Tree/shrub & 1.4 & 27.4 & 0.3 & 0.1 & 0.2 & 0.1 & 0.8 & 30.3 & 1.5 \\
\hline & Imp. bldg & 0.0 & 0.0 & 13.5 & 0.0 & 0.0 & 0.0 & 0.2 & 13.7 & 1.1 \\
\hline & Imp. road & 0.0 & 0.0 & 0.0 & 12.0 & 0.0 & 0.0 & 0.0 & 12.0 & 1.0 \\
\hline & Imp. other & 0.1 & 0.0 & 0.1 & 0.0 & 11.8 & 0.0 & 0.1 & 12.2 & 1.0 \\
\hline & Water & 0.0 & 0.0 & 0.0 & 0.0 & 0.0 & 0.0 & 0.0 & 0.0 & 0.0 \\
\hline & Soil & 0.4 & 0.0 & 0.0 & 0.0 & 0.1 & 0.0 & 1.2 & 1.7 & 0.4 \\
\hline & 2nd year total & 30.6 & 27.4 & 14.4 & 12.1 & 12.7 & 0.3 & 2.6 & & \\
\hline & 2nd year SE & 1.5 & 1.4 & 1.1 & 1.0 & 1.1 & 0.2 & 0.5 & & \\
\hline & Net (2004-2009) & 0.5 & -3.0 & 0.7 & 0.1 & 0.5 & 0.3 & 0.8 & & \\
\hline \multirow[t]{10}{*}{ Kansas City, MO (2003-2009) } & Grass/herb & 48.5 & 0.5 & 0.2 & 0.3 & 0.7 & 0.1 & 0.3 & 50.7 & 1.6 \\
\hline & Tree/shrub & 1.1 & 27.5 & 0.0 & 0.1 & 0.0 & 0.0 & 0.5 & 29.2 & 1.4 \\
\hline & Imp. bldg & 0.0 & 0.0 & 4.6 & 0.0 & 0.2 & 0.0 & 0.0 & 4.8 & 0.7 \\
\hline & Imp. road & 0.0 & 0.0 & 0.0 & 6.3 & 0.0 & 0.0 & 0.0 & 6.3 & 0.8 \\
\hline & Imp. other & 0.0 & 0.0 & 0.2 & 0.1 & 6.8 & 0.0 & 0.0 & 7.1 & 0.8 \\
\hline & Water & 0.0 & 0.0 & 0.0 & 0.0 & 0.0 & 0.0 & 0.0 & 0.0 & 0.0 \\
\hline & Soil & 0.2 & 0.0 & 0.0 & 0.0 & 0.7 & 0.0 & 1.0 & 1.9 & 0.4 \\
\hline & 2nd year total & 49.8 & 28.0 & 5.0 & 6.8 & 8.4 & 0.1 & 1.8 & & \\
\hline & 2nd year SE & 1.6 & 1.4 & 0.7 & 0.8 & 0.9 & 0.1 & 0.4 & & \\
\hline & Net (2003-2009) & -0.8 & -1.2 & 0.2 & 0.5 & 1.3 & 0.1 & -0.1 & & \\
\hline \multirow[t]{10}{*}{ Los Angeles, CA (2005-2009) } & Grass/herb & 21.0 & 0.0 & 0.3 & 0.0 & 0.2 & 0.0 & 0.3 & 21.8 & 1.3 \\
\hline & Tree/shrub & 0.4 & 20.6 & 0.2 & 0.0 & 0.3 & 0.0 & 0.0 & 21.5 & 1.3 \\
\hline & Imp. bldg & 0.0 & 0.0 & 21.0 & 0.0 & 0.2 & 0.0 & 0.0 & 21.2 & 1.3 \\
\hline & Imp. road & 0.0 & 0.0 & 0.0 & 14.7 & 0.0 & 0.0 & 0.0 & 14.7 & 1.1 \\
\hline & Imp. other & 0.0 & 0.0 & 0.4 & 0.0 & 15.8 & 0.0 & 0.1 & 16.3 & 1.2 \\
\hline & Water & 0.0 & 0.0 & 0.0 & 0.0 & 0.0 & 0.0 & 0.0 & 0.0 & 0.0 \\
\hline & Soil & 0.3 & 0.0 & 0.5 & 0.2 & 0.2 & 0.0 & 3.1 & 4.3 & 0.6 \\
\hline & 2nd year total & 21.7 & 20.6 & 22.4 & 14.9 & 16.7 & 0.0 & 3.5 & & \\
\hline & 2nd year SE & 1.3 & 1.3 & 1.3 & 1.1 & 1.2 & 0.0 & 0.6 & & \\
\hline & Net (2005-2009) & -0.1 & -0.9 & 1.2 & 0.2 & 0.4 & 0.0 & -0.8 & & \\
\hline \multirow[t]{10}{*}{ Miami, FL (2003-2009) } & Grass/herb & 14.2 & 0.2 & 0.1 & 0.1 & 0.6 & 0.0 & 0.1 & 15.3 & 1.2 \\
\hline & Tree/shrub & 1.1 & 21.2 & 0.4 & 0.2 & 0.1 & 0.1 & 0.1 & 23.3 & 1.4 \\
\hline & Imp. bldg & 0.3 & 0.0 & 23.5 & 0.0 & 0.0 & 0.0 & 0.1 & 23.9 & 1.4 \\
\hline & Imp. road & 0.0 & 0.0 & 0.0 & 18.0 & 0.0 & 0.0 & 0.0 & 18.0 & 1.3 \\
\hline & Imp. other & 0.2 & 0.2 & 0.6 & 0.0 & 17.0 & 0.0 & 0.1 & 18.1 & 1.3 \\
\hline & Water & 0.0 & 0.0 & 0.0 & 0.0 & 0.0 & 0.0 & 0.0 & 0.0 & 0.0 \\
\hline & Soil & 0.0 & 0.0 & 0.2 & 0.0 & 0.2 & 0.0 & 1.0 & 1.4 & 0.4 \\
\hline & 2nd year total & 15.9 & 21.6 & 24.8 & 18.3 & 17.9 & 0.1 & 1.4 & & \\
\hline & 2nd year SE & 1.2 & 1.4 & 1.4 & 1.3 & 1.3 & 0.1 & 0.4 & & \\
\hline & Net (2003-2009) & 0.6 & -1.7 & 0.9 & 0.3 & -0.2 & 0.1 & 0.0 & & \\
\hline \multirow[t]{10}{*}{ Minneapolis, MN (2003-2008) } & Grass/herb & 18.6 & 0.2 & 0.0 & 0.0 & 0.3 & 0.0 & 0.4 & 19.6 & 1.3 \\
\hline & Tree/shrub & 1.0 & 33.7 & 0.1 & 0.2 & 0.1 & 0.0 & 0.0 & 35.1 & 1.6 \\
\hline & Imp. bldg & 0.0 & 0.0 & 14.4 & 0.0 & 0.1 & 0.0 & 0.1 & 14.6 & 1.2 \\
\hline & Imp. road & 0.0 & 0.1 & 0.0 & 12.3 & 0.0 & 0.0 & 0.0 & 12.4 & 1.1 \\
\hline & Imp. other & 0.0 & 0.0 & 0.3 & 0.0 & 15.6 & 0.0 & 0.0 & 15.9 & 1.2 \\
\hline & Water & 0.0 & 0.0 & 0.0 & 0.0 & 0.0 & 0.0 & 0.0 & 0.0 & 0.0 \\
\hline & Soil & 0.2 & 0.0 & 0.1 & 0.0 & 0.1 & 0.2 & 1.7 & 2.4 & 0.5 \\
\hline & 2nd year total & 19.8 & 34.1 & 14.9 & 12.5 & 16.2 & 0.2 & 2.3 & & \\
\hline & 2nd year SE & 1.3 & 1.6 & 1.2 & 1.1 & 1.2 & 0.2 & 0.5 & & \\
\hline & Net (2003-2008) & 0.2 & -1.1 & 0.3 & 0.1 & 0.3 & 0.2 & -0.1 & & \\
\hline \multirow[t]{8}{*}{ Nashville, TN (2003-2008) } & Grass/herb & 28.3 & 0.4 & 0.1 & 0.1 & 0.0 & 0.0 & 0.3 & 29.2 & 1.4 \\
\hline & Tree/shrub & 0.7 & 49.4 & 0.1 & 0.2 & 0.1 & 0.0 & 0.5 & 51.1 & 1.6 \\
\hline & Imp. bldg & 0.0 & 0.0 & 5.4 & 0.0 & 0.0 & 0.0 & 0.0 & 5.4 & 0.7 \\
\hline & Imp. road & 0.0 & 0.0 & 0.0 & 5.6 & 0.0 & 0.0 & 0.0 & 5.6 & 0.7 \\
\hline & Imp. other & 0.0 & 0.0 & 0.0 & 0.0 & 6.7 & 0.0 & 0.0 & 6.7 & 0.8 \\
\hline & Water & 0.0 & 0.0 & 0.0 & 0.0 & 0.0 & 0.0 & 0.0 & 0.0 & 0.0 \\
\hline & Soil & 0.4 & 0.0 & 0.2 & 0.0 & 0.3 & 0.1 & 0.9 & 1.9 & 0.4 \\
\hline & 2nd year total & 29.4 & 49.8 & 5.8 & 5.9 & 7.2 & 0.1 & 1.7 & & \\
\hline
\end{tabular}


Table 2 (Continued)

\begin{tabular}{|c|c|c|c|c|c|c|c|c|c|c|}
\hline \multirow[t]{2}{*}{ City } & \multirow[t]{2}{*}{1 st year cover class } & \multicolumn{7}{|c|}{2 nd year cover class } & \multicolumn{2}{|c|}{1 st year } \\
\hline & & Grass/herb ${ }^{a}$ & Tree/shrub & Imp. bldg ${ }^{\mathrm{b}}$ & Imp. road ${ }^{c}$ & Imp. other ${ }^{d}$ & Water & Soil & Total & SE \\
\hline & 2nd year SE & 1.4 & 1.6 & 0.7 & 0.8 & 0.8 & 0.1 & 0.4 & & \\
\hline & Net (2003-2008) & 0.2 & -1.2 & 0.4 & 0.3 & 0.4 & 0.1 & -0.2 & & \\
\hline \multirow[t]{10}{*}{ New Orleans, LA (2005-2009) } & Grass/herb & 22.7 & 0.0 & 0.0 & 0.0 & 0.2 & 0.4 & 0.7 & 24.0 & 1.8 \\
\hline & Tree/shrub & 6.6 & 23.3 & 0.0 & 0.9 & 1.1 & 0.4 & 0.7 & 32.9 & 2.0 \\
\hline & Imp. bldg & 1.4 & 0.0 & 14.6 & 0.0 & 0.4 & 0.0 & 0.4 & 16.7 & 1.6 \\
\hline & Imp. road & 0.0 & 0.0 & 0.0 & 15.5 & 0.0 & 0.0 & 0.0 & 15.5 & 1.5 \\
\hline & Imp. other & 0.2 & 0.0 & 0.0 & 0.0 & 9.1 & 0.0 & 0.0 & 9.2 & 1.2 \\
\hline & Water & 0.0 & 0.0 & 0.0 & 0.0 & 0.0 & 0.0 & 0.0 & 0.0 & 0.0 \\
\hline & Soil & 0.2 & 0.0 & 0.0 & 0.0 & 0.2 & 0.0 & 1.4 & 1.8 & 0.6 \\
\hline & 2nd year total & 31.1 & 23.3 & 14.6 & 16.3 & 10.8 & 0.7 & 3.2 & & \\
\hline & 2nd year SE & 2.0 & 1.8 & 1.5 & 1.6 & 1.3 & 0.4 & 0.7 & & \\
\hline & Net (2005-2009) & 7.1 & -9.6 & -2.1 & 0.9 & 1.6 & 0.7 & 1.4 & & \\
\hline \multirow[t]{10}{*}{ New York, NY (2004-2009) } & Grass/herb & 14.9 & 0.1 & 0.2 & 0.0 & 0.6 & 0.1 & 0.6 & 16.6 & 1.2 \\
\hline & Tree/shrub & 1.2 & 19.3 & 0.0 & 0.2 & 0.2 & 0.0 & 0.0 & 20.9 & 1.3 \\
\hline & Imp. bldg & 0.0 & 0.0 & 24.4 & 0.0 & 0.1 & 0.0 & 0.0 & 24.6 & 1.4 \\
\hline & Imp. road & 0.0 & 0.0 & 0.0 & 16.1 & 0.0 & 0.0 & 0.0 & 16.1 & 1.2 \\
\hline & Imp. other & 0.0 & 0.3 & 0.2 & 0.1 & 18.5 & 0.0 & 0.0 & 19.1 & 1.3 \\
\hline & Water & 0.0 & 0.0 & 0.0 & 0.0 & 0.0 & 0.0 & 0.1 & 0.1 & 0.1 \\
\hline & Soil & 0.5 & 0.0 & 0.3 & 0.0 & 0.1 & 0.0 & 1.8 & 2.7 & 0.5 \\
\hline & 2nd year total & 16.6 & 19.7 & 25.2 & 16.4 & 19.5 & 0.1 & 2.5 & & \\
\hline & 2nd year SE & 1.2 & 1.3 & 1.4 & 1.2 & 1.3 & 0.1 & 0.5 & & \\
\hline & Net (2004-2009) & 0.0 & -1.2 & 0.6 & 0.3 & 0.4 & 0.0 & -0.2 & & \\
\hline \multirow[t]{10}{*}{ Pittsburgh, PA (2004-2008) } & Grass/herb & 16.9 & 0.0 & 0.0 & 0.0 & 0.1 & 0.0 & 0.1 & 17.1 & 1.2 \\
\hline & Tree/shrub & 0.2 & 41.6 & 0.1 & 0.0 & 0.0 & 0.0 & 0.0 & 41.9 & 1.6 \\
\hline & Imp. bldg & 0.0 & 0.0 & 14.7 & 0.0 & 0.1 & 0.0 & 0.1 & 14.9 & 1.2 \\
\hline & Imp. road & 0.0 & 0.0 & 0.0 & 13.3 & 0.0 & 0.0 & 0.1 & 13.4 & 1.1 \\
\hline & Imp. other & 0.0 & 0.0 & 0.1 & 0.0 & 11.6 & 0.0 & 0.0 & 11.7 & 1.0 \\
\hline & Water & 0.0 & 0.0 & 0.0 & 0.0 & 0.0 & 0.0 & 0.0 & 0.0 & 0.0 \\
\hline & Soil & 0.1 & 0.0 & 0.0 & 0.0 & 0.0 & 0.0 & 0.8 & 1.0 & 0.3 \\
\hline & 2nd year total & 17.2 & 41.6 & 14.9 & 13.3 & 11.8 & 0.0 & 1.2 & & \\
\hline & 2nd year SE & 1.2 & 1.6 & 1.2 & 1.1 & 1.0 & 0.0 & 0.3 & & \\
\hline & Net (2004-2008) & 0.1 & -0.3 & 0.0 & -0.1 & 0.1 & 0.0 & 0.2 & & \\
\hline \multirow[t]{10}{*}{ Portland, OR (2005-2009) } & Grass/herb & 21.4 & 0.3 & 0.3 & 0.1 & 0.3 & 0.0 & 0.2 & 22.7 & 1.3 \\
\hline & Tree/shrub & 0.7 & 30.4 & 0.1 & 0.0 & 0.3 & 0.0 & 0.0 & 31.5 & 1.5 \\
\hline & Imp. bldg & 0.2 & 0.1 & 14.4 & 0.0 & 0.0 & 0.0 & 0.0 & 14.7 & 1.1 \\
\hline & Imp. road & 0.0 & 0.0 & 0.0 & 12.5 & 0.0 & 0.0 & 0.0 & 12.5 & 1.1 \\
\hline & Imp. other & 0.0 & 0.1 & 0.1 & 0.0 & 15.8 & 0.0 & 0.0 & 16.0 & 1.2 \\
\hline & Water & 0.0 & 0.0 & 0.0 & 0.0 & 0.0 & 0.0 & 0.0 & 0.0 & 0.0 \\
\hline & Soil & 0.6 & 0.0 & 0.2 & 0.1 & 0.5 & 0.0 & 1.2 & 2.6 & 0.5 \\
\hline & 2nd year total & 23.0 & 30.9 & 15.1 & 12.7 & 16.9 & 0.0 & 1.4 & & \\
\hline & 2nd year SE & 1.3 & 1.5 & 1.1 & 1.1 & 1.2 & 0.0 & 0.4 & & \\
\hline & Net (2005-2009) & 0.3 & -0.6 & 0.4 & 0.2 & 0.9 & 0.0 & -1.2 & & \\
\hline \multirow[t]{10}{*}{ Spokane, WA (2002-2007) } & Grass/herb & 24.0 & 0.7 & 0.2 & 0.1 & 0.4 & 0.0 & 1.7 & 27.1 & 1.0 \\
\hline & Tree/shrub & 0.5 & 20.6 & 0.2 & 0.0 & 0.1 & 0.0 & 1.1 & 22.4 & 0.9 \\
\hline & Imp. bldg & 0.0 & 0.0 & 12.0 & 0.0 & 0.1 & 0.0 & 0.0 & 12.1 & 0.7 \\
\hline & Imp. road & 0.0 & 0.0 & 0.0 & 11.1 & 0.0 & 0.0 & 0.1 & 11.1 & 0.7 \\
\hline & Imp. other & 0.1 & 0.0 & 0.0 & 0.0 & 10.5 & 0.0 & 0.1 & 10.6 & 0.7 \\
\hline & Water & 0.1 & 0.1 & 0.0 & 0.0 & 0.0 & 0.0 & 0.0 & 0.1 & 0.1 \\
\hline & Soil & 1.3 & 0.5 & 0.5 & 0.2 & 0.7 & 0.0 & 13.6 & 16.7 & 0.8 \\
\hline & 2nd year total & 25.9 & 21.8 & 12.8 & 11.4 & 11.6 & 0.0 & 16.5 & & \\
\hline & 2nd year SE & 1.0 & 0.9 & 0.8 & 0.7 & 0.7 & 0.0 & 0.8 & & \\
\hline & Net (2002-2007) & -1.2 & -0.6 & 0.8 & 0.3 & 1.0 & -0.1 & -0.2 & & \\
\hline \multirow[t]{10}{*}{ Syracuse, NY (2003-2009)f } & Grass/herb & 21.7 & 1.6 & 0.1 & 0.6 & na & 0.0 & na & 24.1 & 1.4 \\
\hline & Tree/shrub & 0.5 & 25.0 & 0.1 & 0.3 & na & 0.0 & na & 25.9 & 1.4 \\
\hline & Imp. bldg & 0.7 & 0.0 & 18.9 & 0.1 & na & 0.0 & na & 19.7 & 1.3 \\
\hline & Imp. road & 0.6 & 0.3 & 0.2 & 29.2 & na & 0.0 & na & 30.3 & 1.5 \\
\hline & Imp. other & na & na & na & na & na & 0.0 & na & na & na \\
\hline & Water & 0.0 & 0.0 & 0.0 & 0.0 & 0.0 & 0.0 & 0.0 & 0.0 & 0.0 \\
\hline & Soil & na & na & na & na & na & 0.0 & na & na & na \\
\hline & 2nd year total & 23.6 & 26.9 & 19.3 & 30.2 & na & 0.0 & na & & \\
\hline & 2nd year SE & 1.4 & 1.4 & 1.3 & 1.5 & na & 0.0 & na & & \\
\hline & Net (2003-2009) & -0.5 & 1.0 & -0.4 & -0.1 & na & 0.0 & na & & \\
\hline \multirow[t]{2}{*}{ Tacoma, WA (2001-2005) } & Grass/herb & 24.8 & 1.2 & 0.1 & 0.1 & 0.8 & 0.0 & 0.1 & 27.1 & 1.5 \\
\hline & Tree/shrub & 1.8 & 21.3 & 0.1 & 0.0 & 0.8 & 0.0 & 0.4 & 24.4 & 1.4 \\
\hline
\end{tabular}


Table 2 (Continued)

\begin{tabular}{|c|c|c|c|c|c|c|c|c|c|c|}
\hline \multirow[t]{2}{*}{ City } & \multirow[t]{2}{*}{1 st year cover class } & \multicolumn{7}{|c|}{ 2nd year cover class } & \multicolumn{2}{|c|}{ 1st year } \\
\hline & & Grass/herb ${ }^{a}$ & Tree/shrub & Imp. bldg ${ }^{\mathrm{b}}$ & Imp. road ${ }^{c}$ & Imp. other ${ }^{d}$ & Water & Soil & Total & SE \\
\hline & Imp. bldg & 0.2 & 0.0 & 13.2 & 0.0 & 0.3 & 0.0 & 0.2 & 14.0 & 1.1 \\
\hline & Imp. road & 0.0 & 0.0 & 0.0 & 12.5 & 0.0 & 0.0 & 0.0 & 12.5 & 1.1 \\
\hline & Imp. other & 0.0 & 0.1 & 0.1 & 0.1 & 13.8 & 0.0 & 0.1 & 14.2 & 1.2 \\
\hline & Water & 0.0 & 0.0 & 0.0 & 0.0 & 0.0 & 0.0 & 0.0 & 0.0 & 0.0 \\
\hline & Soil & 1.3 & 0.3 & 0.3 & 0.1 & 2.0 & 0.3 & 3.4 & 7.8 & 0.9 \\
\hline & 2nd year total & 28.1 & 23.0 & 13.9 & 12.8 & 17.6 & 0.3 & 4.3 & & \\
\hline & 2nd year SE & 1.5 & 1.4 & 1.1 & 1.1 & 1.3 & 0.2 & 0.7 & & \\
\hline & Net (2001-2005) & 1.0 & -1.4 & -0.1 & 0.3 & 3.4 & 0.3 & -3.5 & & \\
\hline \multirow[t]{10}{*}{ Average 20 cities $^{g}$} & Grass/herb & 23.0 & 0.3 & 0.1 & 0.0 & 0.4 & 0.0 & 0.4 & 24.3 & na \\
\hline & Tree/shrub & 1.1 & 27.8 & 0.2 & 0.1 & 0.3 & 0.0 & 0.4 & 29.9 & na \\
\hline & Imp. bldg & 0.2 & 0.0 & 15.2 & 0.0 & 0.1 & 0.0 & 0.1 & 15.6 & na \\
\hline & Imp. road & 0.0 & 0.0 & 0.0 & 12.1 & 0.0 & 0.0 & 0.0 & 12.1 & na \\
\hline & Imp. other & 0.1 & 0.1 & 0.1 & 0.0 & 13.6 & 0.0 & 0.0 & 13.9 & na \\
\hline & Water & 0.0 & 0.0 & 0.0 & 0.0 & 0.0 & 0.0 & 0.0 & 0.1 & na \\
\hline & Soil & 0.5 & 0.1 & 0.2 & 0.1 & 0.4 & 0.1 & 3.0 & 4.3 & na \\
\hline & 2nd year total & 24.7 & 28.2 & 15.9 & 12.3 & 14.8 & 0.1 & 4.0 & & \\
\hline & 2nd year SE & na & na & na & na & na & na & na & & \\
\hline & Average net & 0.5 & -1.5 & 0.3 & 0.3 & 0.8 & 0.1 & -0.3 & & \\
\hline \multirow[t]{10}{*}{ Average 18 cities $^{\mathrm{h}}$} & Grass/herb & 22.7 & 0.3 & 0.2 & 0.0 & 0.4 & 0.0 & 0.4 & 24.0 & na \\
\hline & Tree/shrub & 0.8 & 28.4 & 0.2 & 0.1 & 0.2 & 0.0 & 0.4 & 30.0 & na \\
\hline & Imp. bldg & 0.1 & 0.0 & 15.1 & 0.0 & 0.1 & 0.0 & 0.1 & 15.4 & na \\
\hline & Imp. road & 0.0 & 0.0 & 0.0 & 11.7 & 0.0 & 0.0 & 0.0 & 11.7 & na \\
\hline & Imp. other & 0.1 & 0.1 & 0.2 & 0.0 & 13.8 & 0.0 & 0.0 & 14.2 & na \\
\hline & Water & 0.0 & 0.0 & 0.0 & 0.0 & 0.0 & 0.0 & 0.0 & 0.1 & na \\
\hline & Soil & 0.5 & 0.1 & 0.2 & 0.1 & 0.4 & 0.1 & 3.2 & 4.6 & na \\
\hline & 2nd year total & 24.2 & 28.8 & 15.9 & 12.0 & 15.0 & 0.1 & 4.2 & & \\
\hline & 2nd year SE & na & na & na & na & na & na & na & & \\
\hline & Average net & 0.1 & -1.1 & 0.4 & 0.2 & 0.8 & 0.0 & -0.4 & & \\
\hline
\end{tabular}

SE - standard error. Net - net difference between the years (2nd year - 1st year).

a Grass and other herbaceous ground cover.

b Impervious cover occupied by buildings.

c Impervious cover occupied by roads.

d Other impervious cover (e.g., sidewalks, driveways, and parking lots).

e Scrub/shrub/chaparral was a cover class only measured in Albuquerque, NM, and is included in tree/shrub cover. This cover class occupied 31.6 percent of the city area in 2006 and dropped to 29.4 percent in 2009, a loss of 2.2 percent of the city area.

$\mathrm{f}$ Soil cover is included in grass and herbaceous cover; impervious other is included in impervious road.

$g$ Results from Syracuse are not included in average of grass/herbaceous, impervious road, impervious other or soil (see table footnote f).

h Average result not including New Orleans or Detroit as these cities were specifically selected due to expected losses from hurricane and emerald ash borer damage respectively. Results from Syracuse are not included in average of grass/herbaceous, impervious road, impervious other or soil (see table footnote f).

Most of the loss of tree cover converted to grass/herbaceous cover ( 47 percent), followed by conversions to impervious cover (29 percent) and bare soil (23 percent) (Table 2). Likewise, new cover most often converted from grass/herbaceous cover (68 percent), followed by impervious cover (17 percent) and bare soil (14 percent). Only one city (Syracuse) exhibited an overall increase in tree cover, with most of this increase coming from grass/herbaceous cover.

Change in percent impervious cover during the varying periods of analysis ranged from an increase of 3.6 percent in Tacoma to a decrease in percent impervious cover of -0.5 in Syracuse (Table 3). Seventeen of the 20 cities analyzed showed an increase in net impervious cover, 16 of those cities had a statistically significant increase. Four cities exhibited small changes in net impervious cover that were not statistically significant from zero (Syracuse, Chicago, Pittsburgh, New Orleans). Percent impervious cover increased on average by 1.4 percent during the varying periods of analysis (1.3 percent for 20 city average) with the greatest increases in percent impervious cover in Tacoma (3.6 percent), Baltimore (2.1 percent) and Kansas City and Spokane (2.0 percent each). The relative increase in impervious cover was as high as 11.2 percent in Kansas City, but averaged 3.9 percent (3.7 percent for 20 city average).
Cities with the greatest annual increase in impervious cover were Los Angeles (average of $550 \mathrm{ha} / \mathrm{yr}$ ), Houston (400 ha/yr) and Albuquerque (280 ha/yr) (Table 3 ). Impervious cover increases per capita were greatest in Tacoma $\left(6.0 \mathrm{~m}^{2} /\right.$ person/yr), Kansas City $\left(5.9 \mathrm{~m}^{2} /\right.$ person $\left./ \mathrm{yr}\right)$ and Albuquerque $\left(5.5 \mathrm{~m}^{2} /\right.$ person/yr) with an average increase of $2.2 \mathrm{~m}^{2} /$ person/yr $\left(2.1 \mathrm{~m}^{2} /\right.$ person/yr for 20 city average). Average annual increase in percent impervious cover was 0.31 percent/yr ( 0.30 percent/yr for 20 city average). Relative annual increase in impervious cover was 0.87 percent $/ y r$ ( 0.82 percent/yr for 20 city average).

The analysis of the 20 cities shows a general loss in tree cover and increase in impervious cover in the mid to late 2000s. This overall trend of change was also exhibited in the results of national urban land cover change using Google Earth imagery. Of the 1000 random paired-points laid throughout the conterminous urban United States, 970 points were interpretable, with average length of time between points of $6.4 \mathrm{yr}$. The most recent imagery had an average year of 2009, but ranged between 2004 and 2011. The older paired image year averaged 2002 with a range of 1990-2006. Tree cover increases between images averaged 2.1 percent $(\mathrm{SE}=0.5$ percent) with average losses of -2.3 percent ( $\mathrm{SE}=0.5$ percent) for an average net change in tree cover of -0.2 percent. Impervious cover increases between images averaged 3.2 percent $(\mathrm{SE}=0.6$ percent) 
Table 3

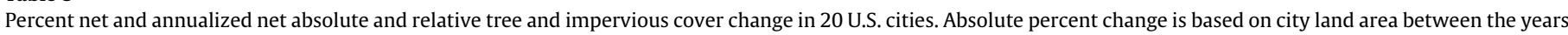

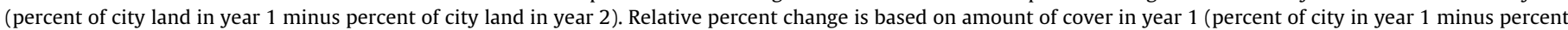

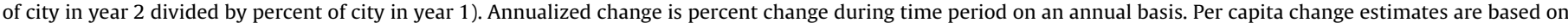
population in the first year of analysis.

\begin{tabular}{|c|c|c|c|c|c|c|c|c|c|c|c|c|}
\hline \multirow[t]{3}{*}{ City } & \multicolumn{4}{|l|}{ Net } & \multicolumn{2}{|c|}{ Tree cover change } & \multicolumn{2}{|c|}{ Impervious cover change } & \multicolumn{4}{|c|}{ Annualized net } \\
\hline & \multicolumn{2}{|c|}{ Absolute change } & \multicolumn{2}{|c|}{ Relative change } & \multirow[t]{2}{*}{$\mathrm{ha} / \mathrm{yr}^{\mathrm{c}}$} & \multirow[t]{2}{*}{$\mathrm{m}^{2} / \mathrm{cap} / \mathrm{yr}^{\mathrm{d}}$} & \multirow[t]{2}{*}{$\mathrm{ha} / \mathrm{yr}^{\mathrm{c}}$} & \multirow[t]{2}{*}{$\mathrm{m}^{2} / \mathrm{cap} / \mathrm{yr}^{\mathrm{d}}$} & \multicolumn{2}{|c|}{ Absolute change } & \multicolumn{2}{|c|}{ Relative change } \\
\hline & Tree $^{\mathrm{a}}$ & Imp. ${ }^{\mathrm{b}}$ & Tree $^{\mathrm{a}}$ & Imp. ${ }^{\mathrm{b}}$ & & & & & Tree $^{a}$ & Imp..$^{b}$ & Tree $^{\mathrm{a}}$ & Imp. ${ }^{b}$ \\
\hline New Orleans, LA (2005-2009) & $-9.6^{* *}$ & 0.4 & $-29.2^{* *}$ & 0.9 & -1120 & -24.6 & 40 & 0.9 & -2.49 & 0.09 & -8.27 & 0.21 \\
\hline Houston, TX (2004-2009) & $-3.0^{* *}$ & $1.3^{* *}$ & $-9.8^{* *}$ & $3.5^{* *}$ & -890 & -4.3 & 400 & 1.9 & -0.60 & 0.26 & -2.03 & 0.69 \\
\hline Albuquerque, NM (2006-2009) & $-2.7^{* *}$ & $1.8^{* *}$ & $-6.6^{* *}$ & $5.1^{* *}$ & -420 & -8.3 & 280 & 5.5 & -0.91 & 0.60 & -2.26 & 1.67 \\
\hline Baltimore, MD (2001-2005) & $-1.9^{* *}$ & $2.1^{* *}$ & $-6.3^{* *}$ & $4.7^{* *}$ & -100 & -1.5 & 110 & 1.7 & -0.48 & 0.51 & -1.62 & 1.16 \\
\hline Atlanta, GA (2005-2009) & $-1.8^{* *}$ & $1.7^{* *}$ & $-3.4^{* *}$ & $6.5^{* *}$ & -150 & -3.1 & 150 & 3.1 & -0.46 & 0.43 & -0.85 & 1.58 \\
\hline Miami, FL (2003-2009) & $-1.7^{* *}$ & $1.0^{*}$ & $-7.1^{* *}$ & $1.7^{*}$ & -30 & -0.8 & 20 & 0.5 & -0.28 & 0.16 & -1.22 & 0.27 \\
\hline Tacoma, WA (2001-2005) & $-1.4^{* *}$ & $3.6^{* *}$ & $-5.8^{* *}$ & $8.9^{* *}$ & -50 & -2.6 & 117 & 6.0 & -0.36 & 0.89 & -1.49 & 2.15 \\
\hline Kansas City, MO (2003-2009) & $-1.2^{* *}$ & $2.0^{* *}$ & $-4.2^{* *}$ & $11.2^{* *}$ & -160 & -3.5 & 270 & 5.9 & -0.20 & 0.34 & -0.71 & 1.78 \\
\hline Nashville, TN (2003-2008) & $-1.2^{* *}$ & $1.1^{* *}$ & $-2.4^{* *}$ & $6.2^{* *}$ & -300 & -5.3 & 270 & 4.8 & -0.24 & 0.22 & -0.48 & 1.21 \\
\hline New York, NY (2004-2009) & $-1.2^{* *}$ & $1.4^{* *}$ & $-5.5^{* *}$ & $2.3^{* *}$ & -180 & -0.2 & 210 & 0.3 & -0.23 & 0.27 & -1.13 & 0.45 \\
\hline Minneapolis, MN (2003-2008) & $-1.1^{* *}$ & $0.8^{* *}$ & $-3.1^{* *}$ & $1.8^{* *}$ & -30 & -0.8 & 20 & 0.5 & -0.22 & 0.15 & -0.63 & 0.35 \\
\hline Boston, MA (2003-2008) & $-0.9^{*}$ & $1.7^{* *}$ & $-3.2^{*}$ & $3.6^{* *}$ & -20 & -0.3 & 40 & 0.7 & -0.19 & 0.35 & -0.65 & 0.71 \\
\hline Los Angeles, CA (2005-2009) & $-0.9^{* *}$ & $1.8^{* *}$ & $-4.2^{* *}$ & $3.4^{* *}$ & -270 & -0.7 & 550 & 1.4 & -0.23 & 0.45 & -1.06 & 0.85 \\
\hline Detroit, MI (2005-2009) & $-0.7^{* *}$ & $1.2^{* *}$ & $-3.0^{* *}$ & $2.6^{* *}$ & -60 & -0.7 & 110 & 1.2 & -0.18 & 0.30 & -0.77 & 0.64 \\
\hline Portland, OR (2005-2009) & -0.6 & $1.5^{* *}$ & -1.9 & $3.5^{* *}$ & -50 & -0.9 & 130 & 2.4 & -0.15 & 0.38 & -0.49 & 0.87 \\
\hline Spokane, WA (2002-2007) & -0.6 & $2.0^{* *}$ & -2.5 & $5.8^{* *}$ & -20 & -1.0 & 60 & 3.0 & -0.11 & 0.39 & -0.50 & 1.14 \\
\hline Chicago, IL (2005-2009) & $-0.5^{* *}$ & 0.0 & $-2.7^{* *}$ & 0.0 & -70 & -0.2 & 0 & 0.0 & -0.13 & 0.00 & -0.69 & 0.00 \\
\hline Pittsburgh, PA (2004-2008) & $-0.3^{*}$ & 0.0 & $-0.8^{*}$ & 0.0 & -10 & -0.3 & 0 & 0.0 & -0.08 & 0.00 & -0.19 & 0.00 \\
\hline Denver, CO (2005-2009) & $-0.3^{*}$ & $1.4^{* *}$ & $-3.1^{*}$ & $3.6^{* *}$ & -30 & -0.5 & 140 & 2.5 & -0.08 & 0.35 & -0.78 & 0.88 \\
\hline Syracuse, NY (2003-2009) & $1.0^{*}$ & -0.5 & $4.0^{*}$ & -1.0 & 10 & 0.7 & -6 & -0.4 & 0.17 & -0.09 & 0.65 & -0.17 \\
\hline 20 city average & -1.5 & 1.3 & -5.0 & 3.7 & & -3.0 & & 2.1 & -0.37 & 0.30 & -1.29 & 0.82 \\
\hline 18 city average $\mathrm{e}^{\mathrm{e}}$ & -1.1 & 1.4 & -3.8 & 3.9 & & -1.9 & & 2.2 & -0.27 & 0.31 & -0.90 & 0.87 \\
\hline
\end{tabular}

a Percent tree and shrub cover (including shrub/scrub/chaparral cover in Albuquerque, NM).

b Percent impervious surfaces (building, roads and other combined).

c Average annual change in hectares per year.

d Average annual change in square meters per capita per year.

e Average result not including New Orleans or Detroit as these cities were specifically selected due to expected losses from hurricane and emerald ash borer damage respectively.

${ }^{*}$ Change significantly different from zero at alpha $=0.90$

** Change significantly different from zero at alpha $=0.95$.

with average losses of -0.4 percent $(\mathrm{SE}=0.2$ percent $)$ for an average net change in impervious cover of +2.8 percent.

\section{Discussion}

While cities expend resources to plant millions of new trees, land development, storms, old age and other factors are reducing the number of older, established trees in cities. Though current planting campaigns may increase tree cover now and in the future, recent trends indicate that tree cover is decreasing in many U.S. cities. Tree cover is decreasing at a rate of about 0.27 percent of the city land area per year, which is equivalent to about 0.9 percent of the existing tree cover being lost annually.

The tree cover loss in the analyzed cities was higher than the average tree cover loss for urban land in the conterminous United States by a factor of about 6 ( $1.1 \mathrm{vs}$. 0.2 percent over the varying time frames). This difference is likely because these analyzed cities do not represent the entire urban area. The selected cities are relatively major cities with increased population densities and likely increased development pressures when compared with the average urban landscape, which includes many smaller, less densely populated areas. These city boundaries, which are often in forested regions, can also include non-urban lands that may have a high likelihood for development and therefore loss of tree cover and increased impervious cover. The change effects in these cities are likely more representative of change in major cities than the national urban change estimates.

Using the national tree cover loss estimate of 0.2 percent of urban land over about a $6 \mathrm{yr}$ period, which equates to about $1 / 30$ of a percent per year, a first order approximation of tree cover loss in urban areas of the conterminous United States is a loss rate of about 7900 ha of urban tree cover per year. Given an average tree density per unit of urban tree cover of approximately 508 trees/ha (average from Cumming et al., 2007; Nowak et al., 2007, in pressa, in press-b; Nowak and Greenfield, 2008; unpublished data), this loss equates to an annual net loss of about 4.0 million trees per year in urban areas of the conterminous United States. This estimate of number of trees lost may be excessive as much of canopy loss may be due to loss of mature trees that would have a lower tree density per unit canopy than the average urban forest, but further research is needed to understand the composition and size class distribution of the canopy loss. Although tree planting and natural regeneration are occurring in urban areas, net tree cover is on a general decline in urban areas of the United States. Tree canopy loss of mature trees, for whatever reason (storms, insects, development, old age), can create relatively large gaps in the canopy cover that will require new tree plantings or regeneration and time to fill.

It is apparent that tree planting and natural regeneration are insufficient to offset the current losses of established urban tree canopies. However, without various tree planting efforts in cities, tree cover loss would be higher. Efforts to facilitate more natural regeneration in cities (e.g., limits on mowing) may also be needed to sustain tree cover. Natural regeneration may not work in all locations (e.g., water limited areas) or produce desired tree species, but it can provide for relatively low cost tree/shrub establishment. Similarly, tree planting may not be appropriate in all cities (e.g., water limited areas) due to the resource costs of maintaining vegetation (e.g., water). Sustaining tree cover not only includes establishing new trees, but also limiting the loss of existing canopy, particularly 
large trees that provide substantial amounts of canopy per tree. Sustaining tree health and protection of healthy tree canopies from human removal (e.g., development) or natural mortality forces (e.g., insects and diseases) can also help sustain existing tree cover and associated environmental services.

Though the current trend is a decline in canopy cover, not all cities are losing tree cover. One of the 20 cities analyzed (Syracuse, $\mathrm{NY}$ ) had an absolute increase in canopy cover of one percent, or 0.2 percent increase per year, with most of the tree cover increase occurring in grass/herbaceous areas. This increase in tree cover matches field data estimates of urban forest change in Syracuse (U.S. Forest Service, unpublished data) that shows that the number of trees (woody plants with stem diameter at $1.37 \mathrm{~m}$ greater than $2.54 \mathrm{~cm}$ ) are increasing. This increase is dominated by European buckthorn (Rhamnus cathartica L.), an invasive small tree/shrub from Europe. Thus, the cover increase in Syracuse is most likely due to natural regeneration in concert with limited development or activities that would tend to reduce regeneration.

New Orleans, as expected, had a significant reduction in tree cover $(-9.6$ percent absolute reduction or -29.2 percent relative reduction), which is most likely due to the devastation of Hurricane Katrina in 2005 (e.g., Chapman et al., 2008). In contrast, the loss in tree cover due to the emerald ash borer in Detroit was lower than expected. Since 2002, this beetle has killed more than 30 million ash trees in Southeastern Michigan (US Forest Service et al., 2011). However, the loss of tree cover in Detroit ( -0.18 percent absolute annual reduction or -0.77 percent relative annual reduction) was less than the average loss from the sampled cities $(-0.27$ percent absolute annual reduction or -0.90 percent relative annual reduction). This difference could be due to ash trees not comprising a major component of overall tree cover in Detroit and/or new trees being established through tree planting programs or natural regeneration that help offset the loss of ash and other trees.

Overall, most of the tree losses converted to grass/herbaceous cover (47 percent) or impervious cover ( 29 percent), while most of the gain of new tree cover also came from grass/herbaceous cover (68 percent) or impervious cover (17 percent). Some of the conversions from tree to impervious cover are due to development, but are also due to impervious cover being beneath trees. When trees are removed, the ground surface beneath the trees switches to the new cover class. Likewise, as trees cover ground surfaces, additional tree cover can tend to reduce impervious cover estimates when trees grow over the impervious surfaces.

Of the overall average increase in impervious cover, about 29 percent of that change was due to changes with loss of tree cover. That 29 percent of newly classified impervious cover is a combination of new development and exposure of existing impervious cover beneath trees. However, at least 71 percent of the impervious cover increase was due to new development. Some cities (i.e., Chicago, Pittsburgh) exhibited no net change in impervious cover during the analysis period, but did exhibit increases and decreases in impervious cover that offset each other. Syracuse exhibited a decrease in impervious cover, which may be, in part, due to the overall increase in tree cover. However, most of the changes in impervious cover in Syracuse occurred with grass/herbaceous cover. New Orleans also lost a substantial amount of building cover (2.1 percent absolute reduction), most likely due to damage from Hurricane Katrina (e.g., Kates et al., 2006).

A better understanding of how tree cover and tree populations are changing can aid managers in developing regeneration or canopy protection plans to sustain adequate tree cover through time and space. Photo-interpretation of paired digital images offers a relatively easy, quick and low-cost means to statistically assess changes among various cover types. To help in quantifying the cover types within an area, a free tool (i-Tree Canopy) is available (www.itreetools.org) that allows users to photo-interpret a city using Google images. This program automatically quantifies the percent cover and associated standard error for each cover class based on user interpretations. Cover data on a city can provide a baseline for developing management plans, setting tree cover goals, and for monitoring change through time. Future analyses on cover distribution or change by land use type or geographic region are needed to investigate patterns and causes of tree and impervious cover changes between and within cities.

The paired digital image analysis offers a relatively quick, easy and cost-effective means to assess cover change, but it does have some limitations. Though Google offers high-resolution imagery in many parts of the world, paired image analysis with Google images is limited by the varying dates among images and varying image resolution. In urban areas, many of the Google images are of sufficient resolution for accurate photo-interpretation and images are continually updated. Obtaining local digital images with known and consistent dates across an area of analysis can overcome the problems associated with varying dates across a study area. Sometimes paired city data also had different image resolution between years, but most images were $1 \mathrm{~m}$ or less. As image interpretation was paired, information from the higher resolution image could aid in interpreting the lower resolution image. Another limitation of the paired image approach is the ability of the interpreter to correctly classify sample points. Interpreter error can lead to inaccurate results, but proper training and testing can produce accurate results. Satellite cover maps also have inherent inaccuracies due to classification errors and can cost tens of thousands of dollars to produce a cover map for a city. The paired photo-interpretation method offers a more cost effective means to assess change, but does not produce a detailed map of cover attributes or cover change across a city.

The results of this study illustrate recent changes in tree and impervious cover in cities and urban areas that can be used to inform planners and policy makers. To determine whether similar trends occurred in the 1990s or early 2000s, and whether these trends will continue in the future, more paired image analyses can be conducted using older paired imagery or by comparing future imagery with contemporary images. More paired image analyses can help better determine both spatial and temporal patterns and rates of landscape cover change. Photo-interpreted data on cover in urban areas and elsewhere can provide an accurate means of assessing cover types and changes in cover through time to help managers and planners make informed decisions on how to better improve local landscapes and the environment.

\section{Conclusion}

Tree cover provides a simple means to assess the magnitude of the overall urban forest and its environmental effects. Despite various and likely limited tree planting and protection campaigns, tree cover tends to be on the decline in U.S. cities while impervious cover is on the increase. While these individual campaigns are helping to increase or reduce the loss of urban tree cover, more widespread, comprehensive and integrated programs that focus on sustaining overall tree canopy may be needed to help reverse the trend of declining tree cover in cities. Net tree cover change is the result of the combined influences of tree planting and natural regeneration, tree growth and tree mortality. Developing coordinated healthy tree canopy programs across various land ownerships can help sustain desired tree cover levels and better manage cover change. Monitoring of tree cover changes is essential to determine current trends and whether desired canopy levels or program effects are being attained. Photo-interpretation of digital aerial images can provide a simple and timely means to assess urban tree cover and how it is changing. 


\section{Acknowledgments}

Funding for this project was provided, in part, by the U.S. Forest Service's RPA Assessment Staff and State \& Private Forestry's Urban and Community Forestry Program and the National Science Foundation (NSF grants DEB-0423476 and BCS-0948952) through the Baltimore Ecosystem Study-Long Term Ecological Research (BES-LTER) and the Syracuse Urban Long-term Research Area Exploratory Award (ULTRA-Ex). The use of trade, firm, or corporation names in this article is for the information and convenience of the reader. Such use does not constitute an official endorsement or approval by the U.S. Department of Agriculture Forest Service of any product or service to the exclusion of others that may be suitable. We thank Mike Boarman, Allison Bodine and Tian Zhou for photo-interpretation. We also thank John Stanovick for his statistical assistance and review, and Mike Galvin and Jackie Lu for their comments on a draft manuscript.

\section{References}

Chapman, E.L., Chambers, J.Q., Ribbeck, K.F., Baker, D.B., Tobler, M.A., Zeng, H., White, D.A., 2008. Hurricane Katrina impacts on forest trees of Louisiana's Pearl River basin. Forest Ecology and Management 256, 883-889.

City of New York, 2011. MillionTreesNYC (retrieved 01.06.11) http://www. milliontreesnyc.org/html/home/home.shtml.

City of Los Angeles, 2011. MillionTreesLA (retrieved 15.06.11) http://www. milliontreesla.org/.

City of Pasadena, 2011. Pasadena Tree Protection Ordinance (retrieved 15.06.11) http://ww2.cityofpasadena.net/publicworks/PNR/TreeOrdinance/default.asp.

City of Seattle, 2011. Seattle's Canopy Cover (retrieved 01.06.11) http://www. seattle.gov/trees/canopycover.html.

Cumming, A.B., Nowak, D.J., Twardus, D.B., Hoehn, R., Mielke, M., Rideout, R., 2007. Urban Forests of Wisconsin 2002: Pilot Monitoring Project 2002. USDA Forest Service, Northeastern Area State and Private Forestry Report, NA-FR-05-07. USDA Forest Service.

Dwyer, J.F., McPherson, E.G., Schroeder, H.W., Rowntree, R.A., 1992. Assessing the benefits and costs of the urban forest. Journal of Arboriculture 18 (5), 227-234. Google Inc., 2011. Google Earth (retrieved 15.01.11) http://earth.google.com.

Hansen, M.C., DeFries, R.S., Townshend, J.R.G., Carroll, M., Dimiceli, C., Sohlberg, R.A., 2003. Global percent tree cover at a spatial resolution of 500 meters: first results of the MODIS vegetation continuous fields algorithm. Earth Interactions 7 (10), $1-15$.

Heisler, G.M., Brazel, A.J., 2010. The urban physical environment: temperature and urban heat islands. In: Aitkenhead-Peterson, J., Volder, A. (Eds.), Urban Ecosystem Ecology (Agronomy Monograph). Soil Science Society of America, Madison, WI, pp. 29-56.

Kates, R.W., Colten, C.E., Laska, S., Leatherman, S.P., 2006. Reconstruction of New Orleans after Hurricane Katrina: a research perspective. PNAS 103 (40), $14653-14660$.

Kuo, F.E., Sullivan, W.C., 2001. Environment and crime in the inner city: does vegetation reduce crime? Environmental Behavior 33 (3), 343-365.

Lindgren, B.W., McElrath, G.W., 1969. Introduction to Probability and Statistics. Macmillan, London.

Lunetta, R.L., Knight, F.K., Ediriwickrema, J., Lyon, J.G., Worthy, L.D., 2006. Land-cover change detection using multi-temporal MODIS NDVI data. Remote Sensing of Environment 105, 142-154.
Maryland Department of Natural Resources, 2011. Cheasapeake Bay Urban Tree Canopy Goals (retrieved 01.10.11) http://www.dnr.state.md.us/forests/ programs/urban/urbantreecanopygoals.asp.

National Research Council, Committee on Hydrologic Impacts of Forest Management, 2008. Hydrologic Effects of a Changing Forest Landscape. The Nationa Academies Press, Washington, DC.

Nowak, D.J., 1993. Historical vegetation change in Oakland and its implications for urban forest management. Journal or Arboriculture 19 (5), 313-319.

Nowak, D.J., Buckelew Cumming, A., Twardus, D., Hoehn, R.E., Brandeis, T.J., Oswalt C.M. Urban Forests of Tennessee. Gen. Tech. Rep. U.S. Department of Agriculture, Forest Service, in press-a.

Nowak, D.J., Buckelew-Cumming, A., Twardus, D., Hoehn, R., Mielke, M., 2007. National Forest Health Monitoring Program, Monitoring Urban Forests in Indiana: Pilot Study 2002. Part 2: Statewide Estimates Using the UFORE Model Northeastern Area Report, NA-FR-01-07.

Nowak, D.J., Dwyer, J.F., 2007. Understanding the benefits and costs of urban forest ecosystems. In: Kuser, J. (Ed.), Urban and Community Forestry in the Northeast. Springer Science and Business Media, New York, pp. 25-46.

Nowak, D.J., Greenfield, E.J., 2008. Urban and Community Forests of New England. USDA Forest Service, Northern Research Station, General Technical Report NRS 38. Newtown Square, PA.

Nowak, D.J., Hoehn, R., Crane, D.E., Bodine, A. Assessing urban forest effects and values in the Great Plains States: Kansas, Nebraska, North Dakota, South Dakota. USDA Forest Service, Northern Research Station, Resource Bulletin NRS, Newtown Square, PA, in press-b.

Nowak, D.J., Rowntree, R.A., McPherson, E.G., Sisinni, S.M., Kerkmann, E., Stevens, J.C., 1996. Measuring and analyzing urban tree cover. Landscape and Urban Planning 36, 49-57.

Oke, T.R., 1989. The micrometeorology of the urban forest. Philosophical Transactions of the Royal Society of London B 324, 335-349.

Parlin, M., 2009. Seattle, Washington Urban Tree Canopy Analysis. NCDC Imaging (retrieved 15.06.11) http://www.seattle.gov/trees/docs/NCDC Final_Project_Report.pdf.

Schwarz, M., Waser, L.T., Zimmerman, N.E., 2006. Change detection based on fractional tree cover derived from MODIS data. In: Kerle, N., Skidmore, A.K. (Eds.), Proceedings of the ISPRS Mid-term Symposium. (retrieved 01.11.11) www.isprs.org/proceedings/XXXVI/Part7/PDF/022.pdf.

Sokal, R.R., Rohlf, F.J., 2003. Biometry: The Principles and Practices of Statistics in Biological Research. W.H. Freeman and Company, New York, NY.

Town of Chapel Hill, 2011. Tree Protection (retrieved 15.06.11) http://www.ci chapel-hill.nc.us/index.aspx?page $=879$.

U.S. Census Bureau, 2007. U.S. Census Data (retrieved 15.01.11) www.census.gov.

U.S. Census Bureau, 2011. Population Estimates (retrieved 01.06.11) http://www. census.gov/popest/cities/SUB-EST2009-4.html.

U.S. Environmental Protection Agency, 1983. Results of the Nationwide Urban Runoff Program: Volume 1 - Final Report. U.S. Environmental Protection Agency, Water Planning Division, Washington, DC. NTIS Accession Number: PB84-185552.

U.S. Environmental Protection Agency, 2011. 2006 National Land Cover Data (NLCD 2006) (retrieved 01.11.11) http://www.epa.gov/mrlc/nlcd-2006.html.

US Forest Service, Michigan State University, Purdue University and Ohio State University, 2011. Emerald Ash Borer, Michigan Information (retrieved 15.06.11) www.emeraldashborer.info/michiganinfo.cfm.

Westphal, L.M., 2003. Urban greening and social benefits: a study of empowerment outcomes. Journal of Arboriculture 29 (3), 137-147.

Wolf, K.M., 2003. Public response to the urban forest in inner-city business districts Journal of Arboriculture 29 (3), 117-126.

Yang, L., Xian, G., Klaver, J.M., Deal, B., 2003. Urban land-cover change detection through sub-pixel imperviousness mapping using remotely sensed data. Photogrammetric Engineering and Remote Sensing 69 (9), 1003-1010.

Zhou, W., Troy, A., Grove, M., 2008. Object-based land cover classification and change analysis in the Baltimore metropolitan area using multitemporal high resolution remote sensing data. Sensors 8, 1613-1636. 